${ }^{1}$ Военно-медищинская академия им. С.М. Кирова (Санкт-Петербург, Россия)

${ }^{2}$ Первый

Санкт-Петербургский государственный медищинский университет им. акад. И.П. Павлова (Санкт-Петербура, Россия)
МОЛЕКУЛЯРНО-БИОЛОГИЧЕСКИЕ АСПЕКТЫ ПРОГНОЗИРОВАНИЯ ОТДАЛЕННЫХ РЕЗУЛЬТАТОВ ХИРУРГИЧЕСКОГО ЛЕЧЕНИЯ МЕСТНО-РАСПРОСТРАНЕННОГО РАКА ТОЛСТОЙ КИШКИ У ПАЦИЕНТОВ СТАРШИХ ВОЗРАСТНЫХ ГРУПП

Г.М. Манихас ${ }^{2}$, Н.А. Майстренко ${ }^{1}$, А.А. Сазонов ${ }^{1}$

\section{MOLECULAR BIOLOGICAL ASPECTS IN PREDICTING \\ THE LONG-TERM RESULTS OF SURGICAL TREATMENT FOR LOCALLY ADVANCED COLORECTAL CANCER IN PATIENTS OF OLDER AGE GROUPS}

Г.М. Манихас ${ }^{2}$

Доктор медищинских наук, профессор, заведуюший кафедрой онкологии, Первый Санкт-Петербургский государственный медииинский университет им. акад. И.П. Павлова, 197022, Санкт-Петербург, ул. Л. Толстого, 6/8.

H.A. Майстренко ${ }^{1}$ Доктор медицинских наук, академик $P A H$, профессор кафедры факультетской хирургии им. С.П. Федорова, Военно-медицинская академия им. С.М. Кирова, 194044, Санкт-Петербург, ул. Академика Лебедева, 6.

A.A. Сазонов ${ }^{1}$

Кандидат медицинских наук, начальник отделения клиники факультетской хирургии им. С.П. Федорова. E-mail: sazonov_alex_doc@mail.ru.

G.M. Manikhas ${ }^{2}$ Doctor of Medicine, Professor, Head of the Department of Oncology Pavlov First St. Petersburg State Medical University, 197022, St. Petersburg, L. Tolstoy str., 6/8.

N.A. Maistrenko ${ }^{1}$ Doctor of Medicine, Academician of the Russian Academy of Sciences, Professor of the Department of Faculty Surgery named after S.P. Fedorov, S.M. Kirov Military Medical Academy, 194044, St. Petersburg, Acad. Lebedeva str., 6.

A.A. Sazonov ${ }^{1}$ Candidate of Medicine, Head of the Department of the Clinic of Faculty Surgery named after S.P. Fedorov E-mail: sazonov_alex_doc@mail.ru.

Цель исследования. Оценка значимости иммуногистохимической диагностики в определении молекулярно-биологических свойств опухоли и прогнозировании результатов хирургического лечения у больных местнораспространенным раком толстой кишки старших возрастных групп.

Материал и методы. Проведена оценка результатов хирургического лечения местно-распространенного рака толстой кишки у пациентов различных 
возрастных групп. Изучена клиническая значимость иммуногистохимических маркеров для прогнозирования отдаленных результатов лечения.

Результаты. Выявлены наиболее значимые факторы риска развития послеоперационных осложнений. Установлена прогностическая значимость таких иммуногистохимических маркеров, как: индекс пролиферативной активности стволовых раковых клеток (ALDH+Ki-67+) и рецептор к хемокинам CXCR4.

Заключение. Применение иммуногистохимической диагностики позволяет получить дополнительную информацию о потенциале злокачественности опухоли, которая необходима для обоснованного выбора хирургической тактики.

Ключевые слова: местно-распространенный рак толстой кишки, иммуногистохимическая диагностика, молекулярный профиль опухоли, пащиенты гериатрического профиля.

Aim. To assess the significance of immunohistochemical diagnostics in determining the molecular biological properties of tumors and predicting the results of surgical treatment in patients with locally advanced colon cancer of older age groups.

Material and methods. The results of surgical treatment of locally advanced colorectal cancer in patients of different age groups were evaluated. The clinical significance of immunohistochemical markers for predicting long-term results was studied.

Results. The most significant risk factors for postoperative complications were identified. The prognostic significance of such immunohistochemical markers as: the index of proliferative activity of cancer stem cells (ALDH+Ki-67+) and the receptor for chemokines CXCR4 was established.

Conclusion. The use of immunohistochemical diagnostics provides additional information about the potential of tumor malignancy, which is necessary for a reasonable choice of surgical tactics.

Keywords: locally advanced colorectal cancer, immunobistochemical diagnosis, molecular profile of the tumor, geriatric patients.

\section{Введение}

$\mathrm{B}$ настоящее время колоректальный рак (КРР) является одним из наиболее распространенных злокачественных новообразований, занимая лидирующие позиции не только по заболеваемости, но и по уровню смертности $[1,2]$. Ежегодно в мире регистрируется около 1,2 миллиона новых случаев КРР и более 600 тысяч человек погибают от этого заболевания [2].

Основной преградой на пути к улучшению результатов лечения КРР является отсутствие эффективных и доступных методик скрининга, что приводит к поздней диагностике неопластического процесса и доминированию распространенных форм данной онкопатологии [3]. Так, местно-распространенный рак (МРР) толстой кишки, выявляемый у 20-30\% больных, не только существенно ограничивает возможности хирургического лечения, но и почти в половине случаев служит основной причиной отказа от выполнения радикальной операции $[4,5]$.

Характерной особенностью злокачественных новообразований толстой кишки является выраженная корреляция частоты их развития с возрастным фактором. Известно, что пик заболеваемости КРР приходится на период 65-70 лет, поэтому данная патология является печальной привилегией лиц пожилого и старческого возраста $[4,6]$. Отягощенность сопутствующими заболеваниями наряду с ограниченными функциональными резервами организма создают существенные трудности для выбора и последующей реализации программ хирургического лечения у этой категории больных. Нельзя игнорировать и возрастные особенности психосоматического статуса у пациентов гериатрического профиля, которые в купе с когнитивными нарушениями зачастую препятствуют адекватному восприятию симптомов. Как следствие, несвоевременная диагностика рака толстой кишки особенно характерна для больных пожилого и старческого возраста, доля которых в структуре распространенных и осложненных форм КРР достигает 80\% [4, 6].

Обобщая приведенные выше факты, следует отметить, что пациенты старших возрастных групп с МРР толстой кишки представляют собой довольно многочисленную и в тоже время наиболее сложную клиническую группу, что подтверждает высокую актуальность проблемы улучшения результатов их лечения. Использование персонифицированного подхода к выбору хирургической тактики является одним из ключевых путей ее решения.

Очевидно, что алгоритм определения рационального объема и вида хирургического вмешательства должен носить комплексный характер и подразумевать анализ показателей функциональной операбельности больного и характеристик опухолевого процесса, что необходимо для прогнозирования эффективности оперативного пособия с позиции как ближайших, так и отдаленных результатов лечения. Необходимо отметить, что используемые в клинической практике морфологические критерии, положенные в основу классификации TNM, далеко не всегда позволяют достоверно прогнозировать результативность тех или иных лечебных программ 
$[7,8]$. Подтверждением этого является существенная неоднородность показателей выживаемости у больных с одинаковой стадией КРР после идентичных по объему вмешательств. Согласно современным представлениям, одним из наиболее перспективных направлений в уточнении прогноза развития онкологического процесса является изучение молекулярно-биологических свойств опухоли с помощью иммуногистохимической (ИГХ) диагностики $[9,10]$.

ИГХ-исследование позволяет получить информацию о концентрации в опухолевых клетках наиболее важных метаболитов, определяющих их потенциал злокачественности на ультраструктурном уровне. В настоящее время ИГХ-диагностика успешно применяется для выбора лечебной тактики при различных новообразованиях, однако сведения об эффективности использования данной методики у больных КРР немногочисленны и противоречивы.

\section{Цель работы}

Оценка значимости ИГХ-диагностики в определении молекулярно-биологических свойств опухоли и прогнозировании результатов хирургического лечения у больных МРР раком толстой кишки старших возрастных групп.

\section{Материал и методы}

Исследование основано на ретроспективном анализе результатов лечения 236 пациентов с мор- фологически верифицированной аденокарциномой толстой кишки, которые были прооперированы в плановом порядке в период с января 2010 по декабрь 2017 года. У всех больных были выявлены местнораспространенные формы КРР без отдаленного метастазирования. На первом этапе исследования производился сравнительный анализ непосредственных и отдаленных результатов хирургического лечения пациентов с учетом возрастного фактора. В рамках второго этапа с помощью ИГХ-диагностики осуществлялся анализ молекулярно-биологических свойств опухоли у пациентов различного возраста, а также оценивалась целесообразность использования отдельных ИГХ-маркеров в прогнозировании отдаленных результатов лечения. Таким образом, в основе настоящей работы лежит сравнительный анализ клинико-морфологических параметров между двумя группами пациентов: старше и моложе 60 лет.

Основная группа была сформирована из 144 больных пожилого и старческого возраста (средний возраст - 71,2 $\pm 3,5$ года). В контрольную группу были включены 92 пациента моложе 60 лет (средний воз-

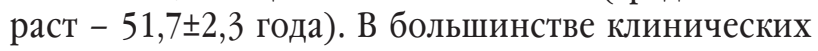
случаев опухоль имела морфологическую структуру умеренно дифференцированной аденокарциномы, при этом, низкая степень дифференцировки в основной группе больных регистрировалась реже, чем в контрольной, однако различия не достигли статистической значимости. Удельный вес больных с метаста-

Клиническая характеристика пациентов

Таблища 1.

\begin{tabular}{|c|c|c|c|}
\hline Признак & $\begin{array}{c}\text { Основная группа, } \\
\text { n=144 } \\
\text { Абс. (\%) }\end{array}$ & $\begin{array}{c}\text { Контрольная группа, } \\
\text { n=92 } \\
\text { Абс. (\%) }\end{array}$ & $\mathbf{P}$ \\
\hline Средний возраст (в годах) & $71,2 \pm 3,5$ & $51,7 \pm 2,3$ & $<0,05$ \\
\hline Соотношение: мужчин/ женщин & $63 / 81(44 / 56)$ & $45 / 47(49 / 51)$ & $>0,05$ \\
\hline $\begin{array}{l}\text { Локализация опухоли: } \\
\text { - прямая кишка } \\
\text { - ободочная кишка }\end{array}$ & $\begin{array}{l}76(53) \\
68(47)\end{array}$ & $\begin{array}{l}51(55) \\
41(45)\end{array}$ & $\begin{array}{l}>0,05 \\
>0,05\end{array}$ \\
\hline $\begin{array}{l}\text { Степень дифференцировки опухоли: } \\
\text { - высокая (G1) } \\
\text { - умеренная (G2) } \\
\text { - низкая (G3) }\end{array}$ & $\begin{array}{c}27(19) \\
101(70) \\
16(11)\end{array}$ & $\begin{array}{l}13(14) \\
61(66) \\
18(20)\end{array}$ & $\begin{array}{l}>0,05 \\
>0,05 \\
>0,05\end{array}$ \\
\hline Регионарные метастазы & $23(16)$ & $21(22)$ & $>0,05$ \\
\hline $\begin{array}{l}\text { Перитуморальные осложнения*: } \\
\text { - нарушение кишечной проходимости } \\
\text { - кровотечение } \\
\text { - абсцесс } \\
\text { - опухолевый свищ } \\
\text { - интоксикация } \\
\text { - распад опухоли }\end{array}$ & $\begin{array}{c}96(67) \\
32(22) \\
14(10) \\
13(9) \\
7(5) \\
6(4)\end{array}$ & $\begin{array}{c}52(57) \\
27(29) \\
4(4) \\
5(5) \\
2(2) \\
2(2)\end{array}$ & $\begin{array}{l}>0,05 \\
>0,05 \\
>0,05 \\
>0,05 \\
>0,05 \\
>0,05\end{array}$ \\
\hline
\end{tabular}

* У 29 пациентов основной группы и у 12 - в контрольной отмечено сочетание из двух и более перитуморальных осложнений. 
Частота вовлечения в опухолевый процесс близлежащих органов и структур

\begin{tabular}{|c|c|c|c|}
\hline Название органа (структуры) & $\begin{array}{c}\text { Основная группа, } \\
\mathrm{n}=144 \\
\text { Абс. (\%) } \\
\end{array}$ & $\begin{array}{c}\text { Контрольная группа, } \\
\mathbf{n}=92 \\
\text { Абс. (\%) } \\
\end{array}$ & $\mathbf{P}$ \\
\hline Матка & $20(14)$ & $16(17)$ & $>0,05$ \\
\hline Влагалище & $18(13)$ & $11(12)$ & $>0,05$ \\
\hline Придатки & $13(9)$ & $11(12)$ & $>0,05$ \\
\hline Семенные пузырьки & $12(8)$ & $10(11)$ & $>0,05$ \\
\hline Простата & $6(4)$ & $5(5)$ & $>0,05$ \\
\hline Мочевой пузырь & $19(13)$ & $9(10)$ & $>0,05$ \\
\hline Мочеточник & $14(10)$ & $6(7)$ & $>0,05$ \\
\hline Тонкая кишка & $22(15)$ & $16(17)$ & $>0,05$ \\
\hline Толстая кишка & $10(7)$ & $4(4)$ & $>0,05$ \\
\hline Желудок & $3(2)$ & $2(2)$ & $>0,05$ \\
\hline Поджелудочная железа & $1(1)$ & $-(0)$ & $>0,05$ \\
\hline Селезенка & $3(2)$ & $2(2)$ & $>0,05$ \\
\hline Большой сальник & $4(3)$ & $5(6)$ & $>0,05$ \\
\hline Наружные подвздошные сосуды & $5(3)$ & $3(3)$ & $>0,05$ \\
\hline Внутр. подвздошные сосуды & $12(8)$ & $6(7)$ & $>0,05$ \\
\hline Ткани брюшной стенки & $17(12)$ & $8(9)$ & $>0,05$ \\
\hline Леваторы & $6(4)$ & $5(6)$ & $>0,05$ \\
\hline Пресакральная фасция & $6(4)$ & $3(3)$ & $>0,05$ \\
\hline
\end{tabular}

* у 36 пациентов основной группы и у 22 - в контрольной отмечено вовлечение в опухолевый процесс 2-х и более органов (структур).

тическим поражением регионарных лимфатических узлов в исследуемых группах был сопоставимым. у большинства пациентов были диагностированы выраженные перитуморальные осложнения, значительно ухудшавшие их общее состояние, при этом, наиболее тяжелые из них чаще регистрировались у больных гериатрического профиля. Таким образом, сравнительная оценка исследуемых возрастных групп по основным характеристикам опухолевого процесса не выявила статистически значимой разницы ни по одному из показателей (табл. 1). Аналогичная закономерность отмечена и при сравнительном анализе частоты вовлечения в неопластический процесс близлежащих структур и органов (табл. 2).

Наличие тех или иных сопутствующих заболеваний было зафиксировано у всех пациентов пожилого и старческого возраста, причем у большинства из них имелось сочетание из нескольких нозологических форм. В контрольной группе сопутствующая патология была выявлена только в 54\% случаев. Высокий уровень полиморбидности у больных пожилого и старческого возраста, наряду с наличием у них более выраженных перитуморальных осложнений, суще- ственно повлияли на распределение пациентов по их физическому статусу, согласно классификации ASA, а также индексу коморбидности Charlson (табл. 3).

Следует отметить, что все пациенты проходили комплексное предоперационное обследование, основными задачами которого было определение распространенности онкологического процесса, резектабельности опухоли, а также оценка функциональных резервов организма. Помимо лабораторно-инструментальных методов, входящих в стандарт обследования хирургического больного, диагностическая программа включала: гастроскопию, эндоскопическое исследование толстой кишки с последующим морфологическим изучением биопсийного материала, УЗИ, МРТ и/или КТ малого таза и брюшной полости. В перечень дополнительных методов обследования входили: гинекологический осмотр, экскреторная урография и цистоскопия при вовлечении в опухолевый процесс мочеполовой системы, КТ грудной клетки для исключения отдаленных метастазов в легких. В случае выявления выраженной сопутствующей патологии привлекались специалисты соответствующего профиля для ком- 
Таблица 3.

Отягощенность больных сопутствующей патологией и их физический статус по шкале ASA

\begin{tabular}{|c|c|c|c|}
\hline Характер сопутствующей патологии & $\begin{array}{c}\text { Основная группа, } \\
\mathbf{n}=144 \\
\text { Абс. (\%) } \\
\end{array}$ & $\begin{array}{c}\text { Контрольная } \\
\text { группа, } \mathrm{n}=92 \\
\text { Абс. }(\%) \\
\end{array}$ & $\mathbf{P}$ \\
\hline Заболевания сердечно-сосудистой системы & $138(96)$ & $51(55)$ & $<0,05$ \\
\hline Заболевания дыхательной системы & $58(40)$ & $17(18)$ & $<0,05$ \\
\hline Заболевания системы пищеварения & $45(31)$ & $24(26)$ & $>0,05$ \\
\hline Заболевания эндокринной системы & $30(21)$ & $3(3)$ & $<0,05$ \\
\hline Заболевания мочеполовой системы & $27(19)$ & $12(13)$ & $>0,05$ \\
\hline Заболевания опорно-двигательной системы & $39(27)$ & $13(13)$ & $>0,05$ \\
\hline Заболевания нервной системы & $16(11)$ & $5(5)$ & $>0,05$ \\
\hline Индекс коморбидности по шкале Charlson >7 & $35(24)$ & $6(6)$ & $<0,05$ \\
\hline $\begin{aligned} \text { Физический статус по шкале ASA: } & \text { - II } \\
& - \text { III } \\
& - \text { IV }\end{aligned}$ & $\begin{array}{c}15(10) \\
112(78) \\
17(12)\end{array}$ & $\begin{array}{l}48(52) \\
41(45) \\
3(3)\end{array}$ & $\begin{array}{l}<0,05 \\
<0,05 \\
<0,05\end{array}$ \\
\hline
\end{tabular}

* у 112 пациентов основной группы и у 31 - в контрольной было зафиксировано по 2 и более сопутствующих заболевания.

плексной оценки степени ее компенсации и влияния на показатели функциональной операбельности. При необходимости проводилась корригирующая терапия с оценкой динамики состояния пациента. Во всех клинических случаях решение о выборе лечебной тактики принималось консилиумом специалистов.

Всем больным были выполнены радикальные оперативные вмешательства, неотъемлемым принципом которых было одномоментное удаление пораженных опухолью органов и тканей единым блоком (en-bloc) с обязательным достижением негативных краев резекции. Кроме того, важным компонентом программы хирургического лечения было использование широкого спектра реконструктивных методик, направленных на сохранение высоких показателей качества жизни больных. Полнота их применения не зависела от возраста. Так, обструктивные резекции толстой кишки выполнялись исключительно редко (менее 3\% случаев для каждой из групп), только при плохой подготовке кишечника, в связи с субкомпенсированным нарушением проходимости. При экзентерациях малого таза у женщин производили сигмовагинопластику, что не только способствовало достижению психосексуальной реабилитации, но и позволяло заполнить свободную полость хорошо васкуляризированной тканью, снизив, тем самым, риск развития инфекционных осложнений. При опухолевой инфильтрации мочевого пузыря без вовлечения треугольника Льето выполняли его резекцию с энтероцистопластикой или формированием континентного ортотопического мочевого резервуара из илеоцекального сегмента кишечника $[2,7]$. С целью закрытия обширных дефектов промежности производили пластику кожно-мышечным лоскутом, выкроенным из верхней порции прямой мышцы живота с сохранением питающей ножки (VRAM лоскут).

Следует отметить, что предоперационная химиолучевая терапия (ХЛТ) и адъювантная полихимиотерапия (ПХТ) чаще проводились у больных контрольной группы, причем, различия в частоте применения ПХТ достигли статистической значимости (рис. 1).

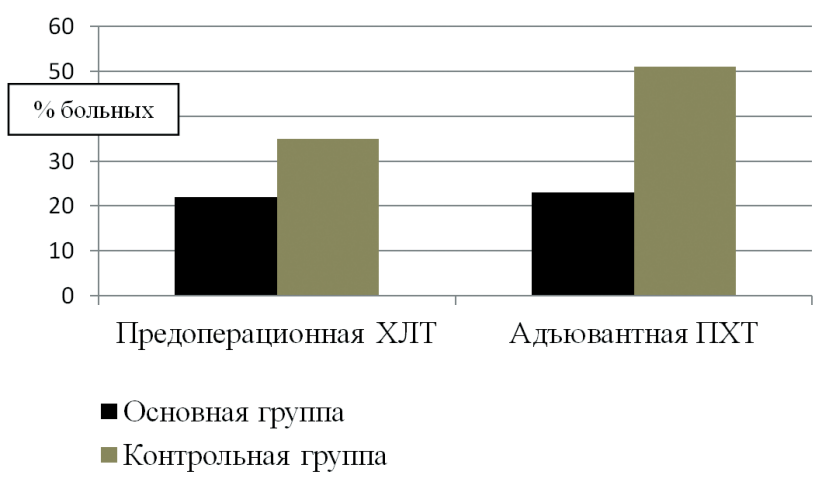

Рис. 1. Частота применения программ колплексного лечения

Иммуногистохимическое (ИГХ) исследование выполнялось для сравнительной оценки молекулярно-биологических свойств аденокарциномы толстой кишки у пациентов различных возрастных групп. Данные ИГХ диагностики сопоставлялись с отдаленными результатами лечения, и на основании дисперсионно-корреляционного анализа определялась клиническая значимость отдельных маркеров в прогнозировании онкологической эффективности хирургического пособия. Для проведения 
ИГХ-исследования был отобран операционный материал 40 пациентов: по 20 препаратов из каждой возрастной группы. На данном материале изучалась общая пролиферативная активность опухоли путем определения уровня экспрессии протеина Ki-67. Также с помощью антител к альдегиддегидрогеназе ALDH1 и методики двойного иммуногистохимического окрашивания оценивалась популяция стволовых раковых клеток и их пролиферативная активность. Кроме того, изучалась экспрессия рецептора к хемокинам CXCR4, который характеризует метастатический потенциал аденокарциномы, индуцируя хемотаксис и инвазию ее клеток в органы мишени.

ИГХ-исследование выполнялось по стандартному протоколу. Из парафиновых блоков изготавливались срезы толщиной 2 микрон с последующим нанесением смеси разведенных антител. Для детекции окрашивания применялась двойная система визуализации DoubleStain в соответствии с прилагаемой инструкцией. Полученные препараты изучались с помощью световой микроскопии.

В ходе выполнения работы применялись современные методы статистического анализа: при сравнении межгрупповых параметров для категориальных значений - точный тест Фишера; для количественных показателей - «t-тест» Стьюдента. Для расчета выживаемости использовали метод Каплан-Майера. При категоризованных переменных различия в группах проверялись с помощью критерия $\chi$-квадрат для таблиц сопряженности. Статистически значимыми считали результаты, при которых значение $p<0,05$. Оценка силы и направления связи между количественными показателями осуществлялась с использованием коэффициента корреляции Пирсона.

Исследование выполнено в соответствии с требованиями Хельсинкской декларации. Все пациенты, участвовавшие в нем, дали письменное согласие.

\section{Результаты}

Оценка ближайших результатов хирургического лечения произведена у всех пациентов, включенных в исследование. Послеоперационные осложнения были зафиксированы у $37 \%$ больных гериатрического профиля и у $28 \%$ пациентов моложе 60 лет $(\mathrm{p}>0,05)$. Сравнительный анализ их структуры показал, что частота развития "собственно хирургических осложнений» (непосредственно связанных с выполнением вмешательства) в обеих группах была сопоставима (табл. 4). В то же время осложнения, «ассоциированные с отягощенностью общесоматического статуса» (возникающие, в первую очередь, вследствие декомпенсации сопутствующей патологии), достоверно чаще встречались у больных гериатрического профиля $(\mathrm{p}<0,05)$. Данная закономерность подтверждает необходимость строгого выполнения всего комплекса мероприятий, направленных на своевременную диагностику и коррекцию сопутствующей патологии у пациентов пожилого и старческого возраста.

В раннем послеоперационном периоде было зафиксировано 4 летальных исхода: 3 - у больных старше 60 лет и 1 - у пациента среднего возраста. Причинами их наступления в основной группе стали: позднее аррозивное профузное послеоперационное кровотечение из крестцового венозного сплетения с развитием геморрагического шока у одного пациента, в двух других случаях смерть наступила вследствие обширного инфаркта миокарда с фибрилляцией желудочков. Причиной единственного летального исхода в контрольной группе стало развитие полиорганной недостаточности на фоне абдоминального сепсиса, вызванного несостоятельностью колоректального анастомоза.

Отдаленные результаты в сроки от 1,5 до 7 лет после хирургического пособия прослежены у 101 пациента гериатрического профиля и у 68 больных молодого и среднего возраста, что составило $70,1 \%$ и 73,9\% от общей численности исследуемых групп. Показатель общей трехлетней выживаемости в основной группе больных оказался незначительно меньше, чем в контрольной: $63,4 \% \pm 0,6$ против $67,6 \% \pm 0,7$, соответственно (рис. 2).

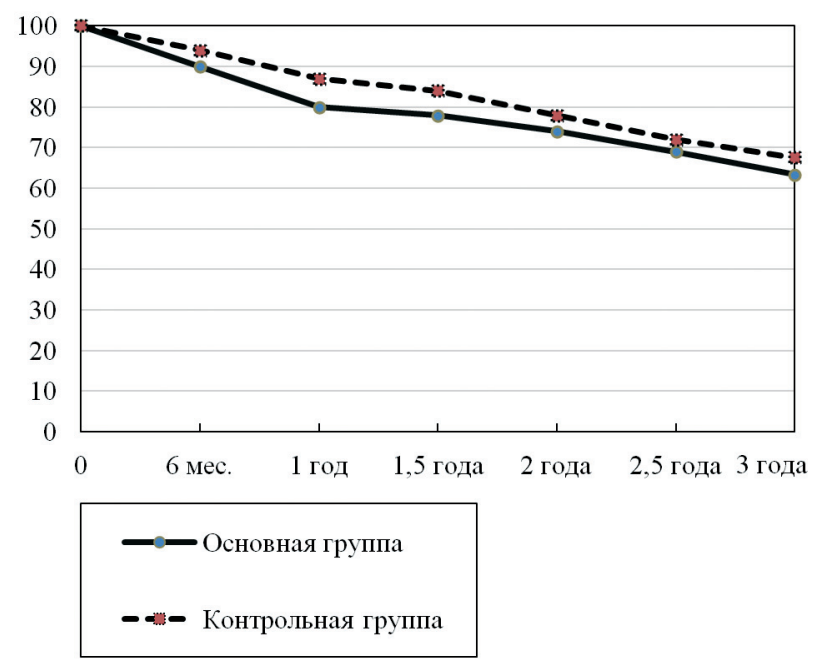

Рис. 2. Общая выживаемость больных за 3-летний период

Безрецидивная трехлетняя выживаемость у пациентов пожилого и старческого возраста, напротив, превзошла таковой показатель среди больных моложе 60 лет: 56,5\% $\pm 0,6$ против 54,4\% $\pm 0,7$, соответственно. Кроме того, необходимо отметить, что канцер-специфическая смертность у больных гериатрического профиля оказалась несколько ниже, чем у пациентов моложе 60 лет: $26,7 \%$ против 30,9\%. Следует отметить, что статистически достоверной разницы между исследуемыми группами при сравнительной оценке приведенных выше показателей не отмечено. Однако была прослежена следующая закономерность: несмотря на меньший уровень общей трехлетней выживаемости у пациентов 
Структура послеоперащионных осложнений

\begin{tabular}{|c|c|c|c|}
\hline Осложнения & $\begin{array}{l}\text { Основная } \\
\text { группа, } \\
\text { Абс. (\%) }\end{array}$ & $\begin{array}{l}\text { Контрольная } \\
\text { группа, } \\
\text { Абс. (\%) }\end{array}$ & $\mathbf{P}$ \\
\hline Собственно хирургические & $39(27 \%)$ & $24(26 \%)$ & $>0,05$ \\
\hline Нагноение послеоперационной раны & $12(8 \%)$ & $5(5 \%)$ & $>0,05$ \\
\hline Лимфорея & $10(7 \%)$ & $5(5 \%)$ & $>0,05$ \\
\hline Эвентрация & $4(3 \%)$ & $2(2 \%)$ & $>0,05$ \\
\hline $\begin{array}{l}\text { Несостоятельность анастомозов: } \\
\text { - колоректальных } \\
\text { - тонко-тонкокишечных }\end{array}$ & $\begin{array}{l}5(6 \%) \\
1(4 \%) \\
\end{array}$ & $\begin{array}{l}2(5 \%) \\
1(6 \%) \\
\end{array}$ & $\begin{array}{l}>0,05 \\
>0,05\end{array}$ \\
\hline $\begin{array}{l}\text { Несостоятельность соустий после реконструкции } \\
\text { мочевыделительной системы }\end{array}$ & $1(6 \%)$ & $1(8 \%)$ & $>0,05$ \\
\hline Ранняя спаечная кишечная непроходимость & $2(1,5 \%)$ & $1(1 \%)$ & $>0,05$ \\
\hline Позднее аррозивное кровотечение & $1(1 \%)$ & $-(0 \%)$ & $>0,05$ \\
\hline Ретракция колостомы & $1(1 \%)$ & $-(0 \%)$ & $>0,05$ \\
\hline Некроз низведенной кишки & $1(1 \%)$ & $1(1 \%)$ & $>0,05$ \\
\hline Перитонит & $5(3 \%)$ & $3(3 \%)$ & $>0,05$ \\
\hline Абсцесс брюшной полости & $1(1 \%)$ & $-(0 \%)$ & $>0,05$ \\
\hline Флегмона малого таза & $1(1 \%)$ & $1(1 \%)$ & $>0,05$ \\
\hline Парез кишечника & $12(8 \%)$ & $8(8 \%)$ & $>0,05$ \\
\hline $\begin{array}{l}\text { Связанные с декомпенсацией } \\
\text { сопутствующей патологии }\end{array}$ & $16(11 \%)$ & $3(3 \%)$ & $<0,05$ \\
\hline Гипостатическая пневмония & $3(2 \%)$ & $-(0 \%)$ & $>0,05$ \\
\hline Острый пиелонефрит & $2(1,5 \%)$ & $1(0 \%)$ & $>0,05$ \\
\hline Кровотечение из хронических язв желудка или ДПК & $2(1,5 \%)$ & $1(1 \%)$ & $>0,05$ \\
\hline Острое нарушение мозгового кровообращения & $2(1,5 \%)$ & $-(0 \%)$ & $>0,05$ \\
\hline Острый инфаркт миокарда & $2(1,5 \%)$ & $-(0 \%)$ & $>0,05$ \\
\hline Тромбоэмболические осложнения & $5(3 \%)$ & $1(1 \%)$ & $>0,05$ \\
\hline
\end{tabular}

* У 18 пациентов основной группы и у 7 - в контрольной отмечено сочетание двух и более осложнений. Частота развития несостоятельности анастомозов и соустий рассчитывалась не от общей численности больных, а от абсолютного числа операций соответствующего профиля.

старше 60 лет, именно в основной группе больных были зафиксированы более благоприятные показатели безрецидивной выживаемости и канцер-специфической летальности. Это обусловлено тем, что почти в 25\% случаев причиной смерти больных гериатрического профиля послужили патологические процессы, не связанные с онкологическим заболеванием. Таким образом, полученные результаты свидетельствуют о высокой онкологической эффективности хирургического лечения больных МРР толстой кишки пожилого и старческого возраста, которая по ряду показателей превзошла таковую для пациентов моложе 60 лет.

Результаты ИГХ-исследования не продемонстрировали влияния индекса пролиферативной активности общего пула клеток аденокарциномы на показатели выживаемости пациентов. Однако при оценке структуры стволовых раковых клеток с помощью методики двойного ИГХ-окрашивания было установлено, что большой удельный вес пролиферирующего клона в их популяции коррелирует с прогрессированием заболевания после хирургического лечения. Так, безрецидивная выживаемость пациентов, у которых доля пролиферирующих клеток в пуле стволовых раковых (ALDH+Ki67+) превышала 30\%, была достоверно меньше. Группа больных, с доминированием непролиферирующих клеток в популяции стволовых раковых (ALDH+Ki67-), напротив, характеризовалась хорошими показателями безрецидивной выживаемости (рис. 3). 

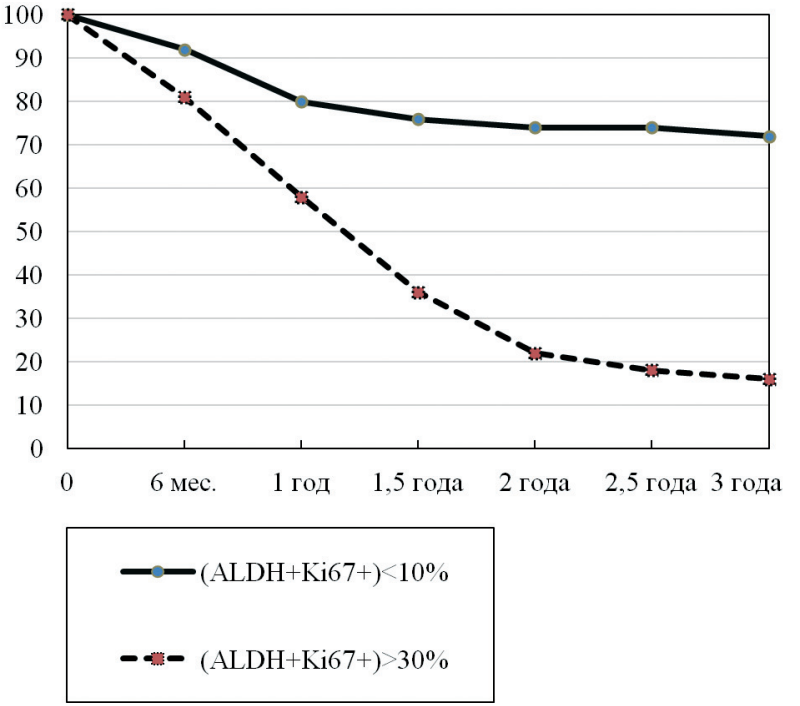

Рис. 3. Безрещидивная выживаемость у больных МРР толстой кишки в зависимости от структуры стволовых раковых клеток

Таким образом, высокий индекс пролиферативной активности стволовых раковых клеток (>30\%) является негативным прогностическим фактором. Кроме того, необходимо отметить, что благоприятный, с точки зрения прогноза отдаленных результатов, иммунофенотип опухоли чаще регистрировался у пациентов гериатрического профиля. Различия между исследуемыми возрастными группами по данному критерию не достигли статистически достоверной разницы ( $\mathrm{p}=0,095)$, что может быть связано с недостаточным числом наблюдений. Однако наличие такой закономерности может служить объяснением достижения обнадеживающих онкологических результатов лечения у пациентов пожилого и старческого возраста.

Важная закономерность была прослежена и при изучении рецептора к хемокинам CXCR4. Высокий уровень экспрессии данного ИГХ-маркера (более, чем в 50\% опухолевых клеток) коррелировал с прогрессированием заболевания (рис. 4). Медиана безрецидивной выживаемости у больных с таким иммунофенотипом аденокарциномы составила всего лишь 14 месяцев. При сравнительном анализе уровня экспрессии рецептора к хемокинам у пациентов различных возрастных групп статистически достоверной разницы между ними по данному критерию выявлено не было $(\mathrm{p}=0,4)$.

Наиболее сильная взаимосвязь иммунофенотипа опухоли и отдаленных результатов лечения была зафиксирована при оценке совокупного влияния структуры стволовых раковых клеток и рецептора к хемокинам CXCR4 (рис. 5). Так, доминирование непролифирирующих клеток в популяции стволовых раковых в сочетании с низкой экспрессией CXCR4 было связано с высоким уровнем безрецидивной выживаемости, медиана которой не была достигнута за

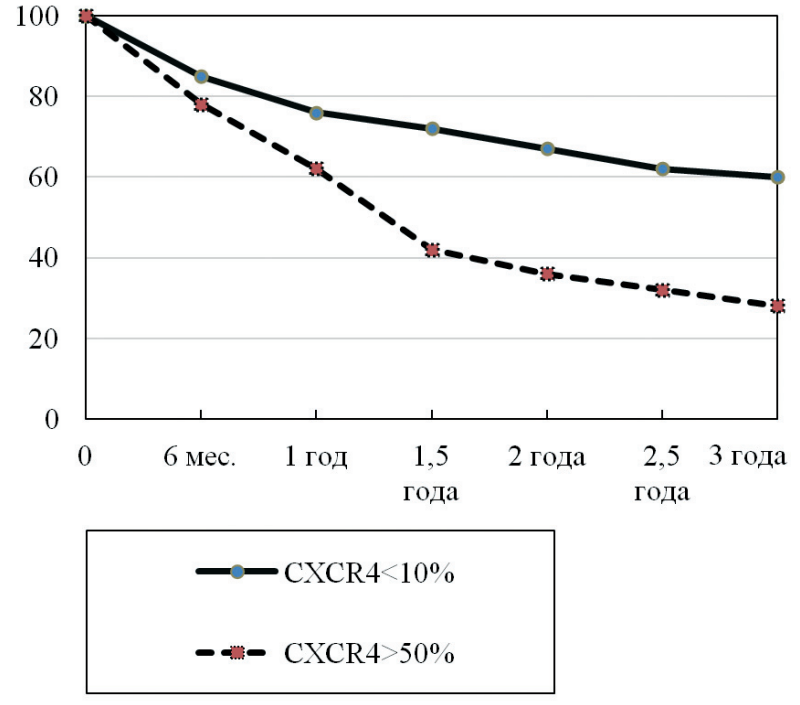

Рис. 4. Безрецидивная выживаемость у больных МРР толстой кишки в зависилости от экспрессии рещептора к хемокинам

трехлетний период наблюдения. В группе пациентов с индексом CXCR4>50\% и долей пролиферирующих раковых клеток >30\%, напротив, наблюдались крайне низкие показатели безрецидивной выживаемости, медиана которой составила всего 9 месяцев.

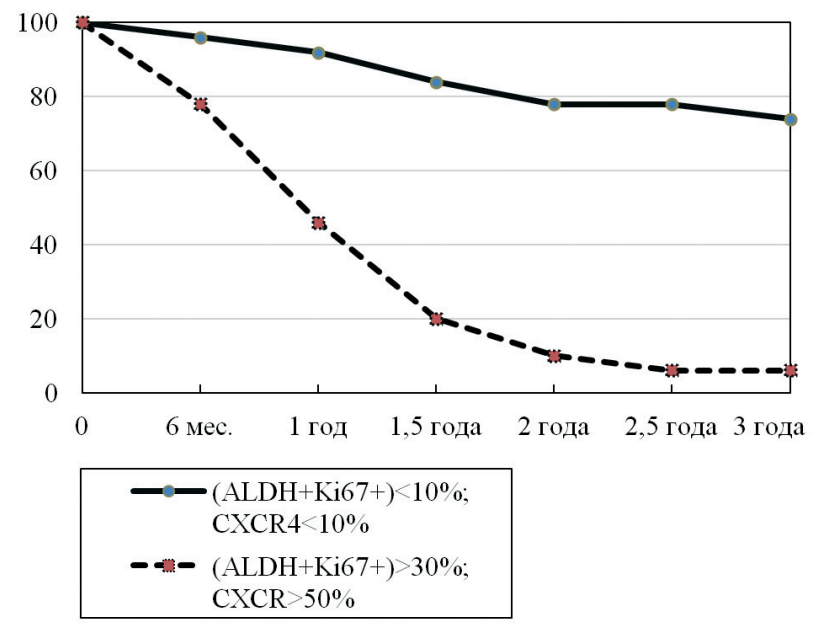

Рис. 5. Безрецидивная выживаемость у больных МРР толстой кишки в зависимости от структуры стволовых раковых клеток и экспрессии СХCR4

\section{Обсуждение}

Хирургическое пособие является основным компонентом лечебной программы при МРР толстой кишки. Для пациентов гериатрического профиля его роль особенно велика, учитывая ограниченные возможности для использования дополнительных методов противоопухолевой терапии (ХЛТ и ПХТ). Собственный клинический опыт наряду с данными других авторов свидетельствуют о преобладании среди больных старших возрастных групп лиц с выра- 
женной сопутствующей патологией и ограниченными функциональными резервами организма, что служит серьезным препятствием для реализации активной хирургической тактики [6]. Нельзя игнорировать и низкий уровень ожидаемой продолжительности жизни у этой категории пациентов. Вместе с тем, эти обстоятельства не должны приводить к отказу от выполнения расширенно-комбинированных вмешательств, однако их следует учитывать при выборе лечебной тактики.

Полученные данные указывают на возможность достижения благоприятных результатов хирургического лечения МРР толстой кишки у пациентов пожилого и старческого возраста при соблюдении персонифицированного подхода к выбору лечебно-диагностических алгоритмов. Одним из путей его реализации наряду с оценкой функциональной операбельности и мероприятиями, направленными на своевременную коррекцию сопутствующей патологии и профилактику послеоперационных осложнений, является изучение иммунофенотипа опухоли.

Приведенные выше результаты свидетельствуют о том, что применение ИГХ-диагностики позволяет получить важные сведенья о молекулярно-биологических свойствах опухолевого процесса, определяющих прогноз заболевания. В частности, установлена высокая прогностическая значимость пролиферативной активности стволовых раковых клеток. Следует отметить, что в настоящее время возрос интерес к изучению этой клеточной культуры, поскольку согласно иерархической теории канцерогенеза именно она играет ключевую роль в развитии и прогрессировании неопластического процесса $[8,9]$. Вместе с тем, в работах, посвященных данной проблеме, внимание акцентируется на определении общей популяции стволовых раковых клеток [9]. Однако полученные нами данные свидетельствуют о прогностической значимости структуры стволовых раковых клеток с позиции их пролиферативной активности.

Важную роль в оценке иммунофенотипа аденокарциномы толстой кишки играет и определение рецептора к хемокинам CXCR4, который, по мнению ряда авторов, отражает метастатический потенциал опухоли $[7,8]$. Результаты настоящей работы подтверждают прогностическую значимость этого ИГХ-маркера, высокий уровень экспрессии которого ассоциируется с неудовлетворительными показателями безрецидивной выживаемости.

\section{Заключение}

Резюмируя вышеизложенное необходимо отметить, что хирургическое лечение больных МРP толстой кишки гериатрического профиля сопровождается повышенным риском развития осложнений, связанных с декомпенсацией сопутствующей патологии, но при этом обеспечивает хорошие отдаленные результаты. Важным направлением в реализации положительного потенциала комбинированных вмешательств у этой категории пациентов может стать применение ИГХ-диагностики, позволяющей получить дополнительную информацию о потенциале злокачественности опухоли, которая необходима для обоснованного выбора хирургической тактики. Подтверждением этого служат результаты проведенного исследования, которые свидетельствуют о прогностической значимости таких ИГХ-маркеров, как: индекс пролиферативной активности стволовых раковых клеток (ALDH+Ki-67+) и рецептор к хемокинам CXCR4, уровень экспрессии которых коррелирует с отдаленными результатами хирургического лечения.

\section{Список литературы}

1. Каприн А.Д., Старинский В.В., Петрова Г.В. Злокачественные новообразования в России в 2016 году // М.: МНИОИ им. П.А. Герцена. - 2017. - 250 с.

2. Ferlay J., Soerjomataram I., Dikshit $R$. et al. Cancer incidence and mortality worldwide: sources, methods and major patterns in GLOBOCAN 2012 // Int J Cancer. - 2015. - №1. - P. 359-386.

3. Куликов Е.П., Бубликов И.Д., Головкин Е.Ю., Семионкин Е.И. Различные хирургические подходы к лечению больных распространенным раком толстой кишки // Онкохирургия. - 2011. - Т. 3, №5. - С. 29-32.

4. Майстренко Н.А., Хватов А.А., Сазонов А.А. Хирургическое лечение больных пожилого и старческого возраста с местно-распространенным раком толстой кишки // Вестник хирургии. - 2016. - Т. 175, №2. - С. $24-32$.

5. Derici $H$. Multivisceral resections for locally advanced rectal cancer // Colorectal Dis. - 2008. - Vol. 10, №5. P. $453-459$.

6. Audisio R.A., Papamichael D. Treatment of colorectal cancer in older patients // Nat Rev Gastroenterol Hepatol. 2012. - Vol. 9, №12. - P. 716-725.

7. Brabletz T., Jung A., Reu S. et al. Variable $\beta$-catenin expression in colorectal cancers indicates tumor progression driven by the tumor environment // Proc Nat Acad Sci USA. - 2012. - Vol. 98. - P. 10356-10361.

8. Пальчева Е.М., Самофбалова О.Ю., Горбачева Ю.В., Царьков П.В. Экспрессия ряда молекулярно-биологических маркеров в первичных аденокарциномах толстой кишки и их метастазах в лимфатических узлах // Архив патологии. - 2012. - №4. - С. 12-18. 
9. Раскин Г.А., Пожарисский К.М., Орлова Р.В. Морфологическая оценка предсказательных факторов при аденокарциноме толстой кишки // Вопросы онкологии. - 2014. - №5. - С. 619-624.

10. Brierley J.D. TNM Classification of Malignant Tumors // ed. J.D. Brierley, M.K. Gospodarowicz, Ch. Wittekind. New York: Wiley Blackwell. - 2017. - 208 p.

\section{References}

1. Kaprin A.D., Starinskij V.V., Petrova G.V. Malignant neoplasms in Russia in 2016. Moscow. Herzen Moscow Scientific-Research Institute. 2017. 250. (In Russ)

2. Ferlay J., Soerjomataram I., Dikshit R. et al. Cancer incidence and mortality worldwide: sources, methods and major patterns in GLOBOCAN 2012. Int J Cancer. 2015; 1(1): 359-386. doi: 10.1002/ijc.29210.

3. Kulikov E.P., Bublikov I.D., Golovkin E.Y., Semionkin E.I. Various surgical approaches to the treatment of patients with advanced colon cancer. Oncosurgery. 2011 Nov; 3(5): 29-32. (In Russ)

4. Maistrenko N.A., Hvatov A.A., Sazonov A.A. Surgical treatment of elderly and senile patients with locally advanced colon cancer. Bulletin of Surgery. 2016 Mar; 175(2): 24-32. (In Russ)

5. Derici $\mathrm{H}$. Multivisceral resections for locally advanced rectal cancer. Colorectal Dis. 2008 Jun; 10(5): 453-459. doi: 10.1111/j.1463-1318.2007.01427.x.

6. Audisio R.A., Papamichael D. Treatment of colorectal cancer in older patients. Nat Rev Gastroenterol Hepatol. 2012 Dec; 9(12): 716-725. doi: 10.1038/nrgastro.2012.196.

7. Brabletz T., Jung A., Reu S. et al. Variable $\beta$-catenin expression in colorectal cancers indicates tumor progression driven by the tumor environment. Proc Nat Acad Sci USA. 2012 Nov; 98(11): 10356-10361. doi: 10.1073/pnas.171610498.

8. Palceva E.M., Samofalova O.Y., Gorbacheva Y.V., Carkov P.V. Expression of a number of molecular biological markers in primary adenocarcinomas of the colon and their lymph node metastases. Archives of Pathology. 2012 Nov; 18(4): 12-18. (In Russ)

9. Raskin G.A., Pozharisskij K.M., Orlova R.V. Morphological assessment of predictive factors in colon adenocarcinoma. Questions of Oncology. 2014 Sep; 12(5): 619-624. (In Russ)

10. Brierley J.D. TNM Classification of Malignant Tumors. ed. J.D. Brierley, M.K. Gospodarowicz, Ch. Wittekind. NewYork: Wiley Blackwell. 2017: 208. 